\title{
Oscillating foils for ship propulsion
}

\author{
J. Mattheijssens ${ }^{1}$, J.-P. Marcel ${ }^{1}$, W. Bosschaerts ${ }^{1}$ \& D. Lefeber ${ }^{2}$ \\ ${ }^{1}$ Department of Mechanics, Royal Military Academy, Belgium \\ ${ }^{2}$ Robotics and Multibody Mechanics Group, \\ Vrije Universiteit Brussel, Belgium
}

\begin{abstract}
The high propulsive efficiency, the fast manoeuvrability and the low noise production of the propulsion of marine animals inspired the development of a new ship propeller. This text describes the design of a flapping foil ship propeller and the experiments performed on it. The flapping foil propeller mimics the tail fin of fish that swim at high speed, like tunas or sharks, in at least two ways: the hydrodynamics and the resonant driving mechanism. The motion of the foil is a combination of a heaving and a pitching oscillation, with a phase difference. The wake behind the tail of a fish has a special structure called the reversed von Karman street. If the motion parameters are well chosen, the wake behind the flapping foil has a similar structure, resulting in positive thrust force and high propulsive efficiency. The driving mechanism uses flexibility to exclude the need for one of the two actuators. The influence of the free surface and the oscillation frequency on the performance are investigated.
\end{abstract}

Keywords: biomimetics, swimming, oscillating foil, ship propulsion.

\section{Introduction}

During millions of years of evolution, fish and marine mammals have developed remarkable propulsion strategies, that have fascinated biologists as well as fluid dynamicists. Depending on the environment of the species and limited by physical and genetical constraints, a wide range of mechanisms can now be observed. Webb classifies all swimming vertebrae in four classes [1]. Class A uses body and/or caudal fins (BCF) for periodic propulsion and is best suited for long-term swimming at relatively high speeds. Class B uses body and/or caudal fins for transient propulsion, well suited for quick starts and turns. The bodies of members 
of this class are flexible and have a large tail area. Class $\mathrm{C}$ uses median and/or paired fins (MPF) for slow swimming and precise manoeuvring, and has better efficiency at low speeds. Members of class D swim only rarely, and are of little interest to our study.

The BCF swimming fish are further divided into subclasses, depending on the fraction of work done by the body or the tail fin. The larger the contribution of the body, the more undulatory the motion. In ostraciiform swimming, only the tail fin flaps from side to side. In anguilliform, a running wave passes through the body, in backward sense. Carangiform swimming is the intermediate mode [2,3].

Because of its good propulsive efficiency at high speeds [4], carangiform swimming was chosen as the inspiration for the development of a new type of ship propulsor. The motion of the tail fin is simplified to the combination of a heaving and a pitching motion.

Experiments in two and three dimensions on rigid, heaving and/or pitching foils in air and water, have shown that these foils can have high efficiency and thrust if the motion parameters are well chosen.

Piziali studied the dynamic stall phenomenon by performing a long series of pressure distribution measurements on foils that performed a small pitching motion around an angle of attack [5]. Three-dimensional effects were studied on a rectangular semispan foil.

Lai and Platzer visualized the flow in a water tunnel around a heaving foil using a dye and performed Laser Doppler Velocimetry on it. One of their main conclusions is that a minimal heaving speed is needed to produce thrust [6].

Triantafyllou and his coworkers studied several types of oscillating foils under different conditions. They identified values for the different motion parameters that ensure good propulsive characteristics, and gained knowledge about the wake structure [7-9].

Lee and Gerontakos studied the boundary layer of a pitching airfoil with an array of hot-film sensors, pressure transducers and smoke visualizations. A detailed description of the evolution of the leading and trailing edge vortices for different conditions was made [10].

Heathcote and Wang and Gursul performed force measurements and Particle Image Velocimetry (PIV) on spanwise flexible, heaving foils. A limited flexibility increased the thrust and the efficiency, but too flexible foils had worse performance [11].

After studying swimming fish for years, Lauder and his coworkers started using oscillating foils in their research. A deformable foil inspired by the pectoral fin and a pair of oscillating foils mimicking the hydrodynamic interaction of the dorsal and caudal fin were developed and tested with PIV and force measurements [12].

Bandyopadhyay, Beal and Menozzi performed force measurements on rolling and pitching foils with different aspect ratios in a tow tank. They also developed an algorithm for fast optimization of the motion parameters [13].

Literature on three-dimensional, heaving and pitching foils was not found, but experiments on three-dimensional foils performing either heaving or pitching, show similar phenomena of fluid dynamics $[11,14]$. 
In the current design, two new features in comparison to earlier designs are present: (1) the hydrofoil oscillates near the water surface and (2) the pitching motion is not driven, but happens passively. The clearance under the ship's keel is often small, and therefore the foil is laid horizontally behind the stern. This way, the foil can have a large aspect ratio, reducing tip losses. The presence of the near surface has unknown effects on the performance of an oscillating foil. The heaving motion is driven, while the pitching motion happens passively under the influence of the hydrodynamic forces. The advantage is that there is no need for an actuator for the pitching motion. The foil will also react passively to non-uniformities in the incoming flow, just the way the flexible fins of fish do.

This paper presents the results of a series of force measurements on a horizontal, heaving and passively-pitching hydrofoil, near the free surface in a still water tank.

\section{Experimental setup and methods}

The desired motion is a combination of heaving and pitching. The heaving motion is a sinusoidally varying vertical displacement of the foil. The pitching motion is a sinusoidally varying rotational angle of the foil around a spanwise axis. Fig. 1 illustrates these motions.

The foil is a rectangular NACA0012 profile with a chord length of $50 \mathrm{~mm}$ and a span of $200 \mathrm{~mm}$. The heaving motion is driven by a motor and a Scotch yoke mechanism, as depicted in Fig. 2. At constant motor speeds, the heaving motion of the slider $h(t)$ is perfectly sinusoidal.

$$
h(t)=h_{0} \sin (2 \pi f t)
$$

with $h_{0}$ the heaving amplitude, which is equal to the crank length and $f$ the heaving frequency, which is equal to the motor rotations per second.

For good propulsive characteristics, the pitch angle should vary sinusoidally in time, with a phase lag of about $90^{\circ}$ to the heaving motion. Fortunately, the heaving motion raises a hydrodynamic moment on the fin, that varies almost like this, and is used to drive the pitching motion. Figure 3 shows the passive pitching mechanism. The foil rotates around an axis parallel to its span. The rotational point has to be upstream enough to make sure the hydrodynamic moment works in the right way, and it has to be higher than the center of mass of the foil to stabilize it. Its position is slightly above the leading edge. The foil is balanced by a counterweight, so

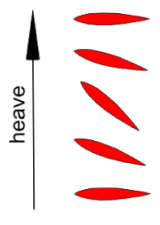

upstroke

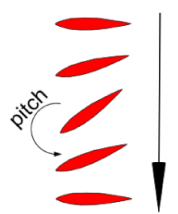

downstroke

Figure 1: Heaving and pitching motion of a foil. 


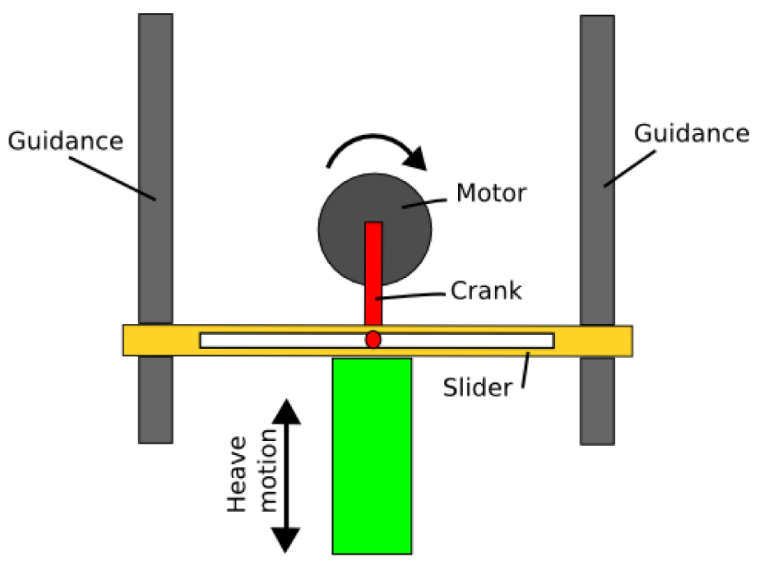

Figure 2: Schematics of the heaving mechanism (Scotch yoke).

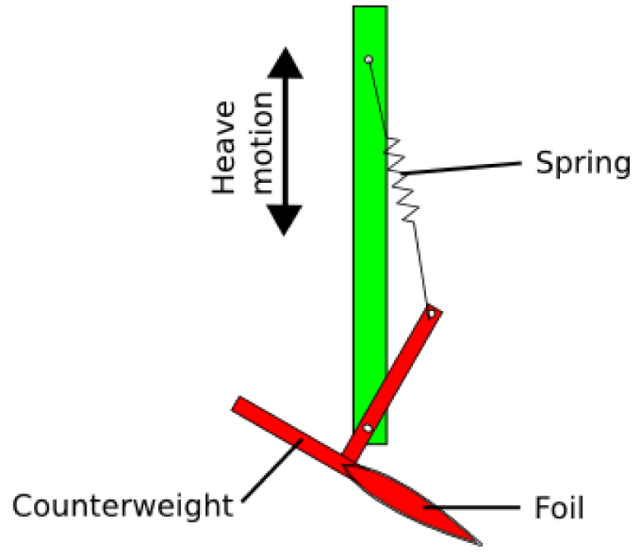

Figure 3: Schematics of the passive pitching mechanism.

that in unloaded condition in water, it is horizontal. If we would let the foil rotate freely, and if we assume that the moment of inertia is negligible, the foil would always rotate until the total hydrodynamic force goes through the rotational point. This would mean a negative thrust. Hence, to counteract the hydrodynamic motion, an eccentric spring is added. The spring pretension can be regulated manually by means of a worm gear.

The motor is a $200 \mathrm{~W}$ brushless Maxon EC-powermax motor, driven with an EPOS2 controller and supplier software on a PC. Two pairs of $120 \Omega$ waterproof strain gauges are attached to and around a flexible structure that is mounted between the heaving and the pitching mechanism. Each pair of gauges facing each other is coupled through a half-bridge for temperature compensation. The two signals of the half-bridges are processed to the two force components: one parallel (thrust) and one perpendicular (lift) to the foil in its neutral position. 
The analog signals of the half-bridges are amplified and digitized by a National Instruments USB-6216 data acquisition system, and transmitted to the PC through a USB interface. The data are logged by a Virtual Instrument written in LabView, and processed and visualized by a program written in Octave. At least 20 motion cycles where logged for each run.

The parameters of the experiments are the frequency $f$ and the nondimensional depth

$$
d=D / c
$$

where $\mathrm{D}$ is the absolute depth of the foil at its highest position in the oscillation cycle. Since the inflow speed is zero, the Strouhal number cannot be defined, so absolute frequencies are used instead. The thrust and lift coefficients are defined as

$$
\begin{aligned}
c_{T} & =\frac{T}{\frac{1}{2} \rho \bar{U}_{h}^{2} c s} \\
c_{L} & =\frac{L}{\frac{1}{2} \rho \bar{U}_{h}^{2} c s}
\end{aligned}
$$

with $T$ and $L$ respectively the thrust and lift, $\rho$ the water density, $\bar{U}_{h}$ the average heaving speed of the foil over all experiments and $c$ and $s$ respectively the chord length and the span of the submerged part of the foil.

The still water tank is a basin of $1200 \mathrm{~mm}$ length (parallel to the foil in its initial position), $1000 \mathrm{~mm}$ width and $800 \mathrm{~mm}$ depth.

The heave amplitude is one chord length. The vertical distance from the leading edge to the rotational point is half a chord length. The distance between the attachment points of the spring is three chord lengths. The spring has an unloaded length of $25 \mathrm{~mm}$ and a stiffness of $0.048 \mathrm{~N} / \mathrm{mm}$. The pretension is kept constant at $0.3 \mathrm{~N}$. From previous experiments on vertical foils, this is known to yield high thrust and efficiency.

The heave frequency range is 0.6 to $1.4 \mathrm{~Hz}$ with $0.1 \mathrm{~Hz}$ increments. The depth, as defined in eqn (2) ranges from zero to two chord lengths, with half chord length increments. This results in 45 runs.

The force coefficients are averaged over at least 20 recorded motion cycles. The efficiency is defined as the average of the ratio of the thrust to the absolute lift:

$$
\nu=\frac{1}{T} \int_{0}^{T} \frac{c_{T}(t)}{\left|c_{L}(t)\right|} d t
$$

A second-degree polynomial least-squares surface was fitted through the data to smoothen outliers away. This surface has equation

$$
z(f, d)=\beta_{0}+\beta_{1} f+\beta_{2} d+\beta_{3} f^{2}+\beta_{4} d^{2}+\beta_{5} f d
$$


where $z$ can mean $c_{t}, c_{L}$ or $\nu$. The parameters $\beta_{i}$ are found using the ordinary least squares, i.e. minimizing the sum of the squares of the errors

$$
\sum_{i=1}^{n} \epsilon_{i}^{2}=\sum_{i=1}^{n}\left(z_{i}-\left(\beta_{0}+\beta_{1} f_{i}+\beta_{2} d_{i}+\beta_{3} f_{i}^{2}+\beta_{4} d_{i}^{2}+\beta_{5} f_{i} d_{i}\right)\right)^{2}
$$

with $n$ the number of measurements. The methodology is described in [15].

\section{Results and discussion}

It is most interesting to observe the trends of the thrust and lift coefficients and the efficiency for the whole parameter domain. This can be done graphically, by studying the contourplots for these quantities in figures 4, 5 and 6 . As can be seen, the highest thrust and lift coefficients occur at average frequencies. What is even more interesting is that at small water depths both coefficients are higher than in deeper water. Since this trend is stronger for the lift than for the thrust, the highest efficiency can be found at an average frequency and a depth around half to one chord length. The average lift coefficient becomes very negative in this zone, meaning a lift force that is mainly upward (our y-axis points downward), and

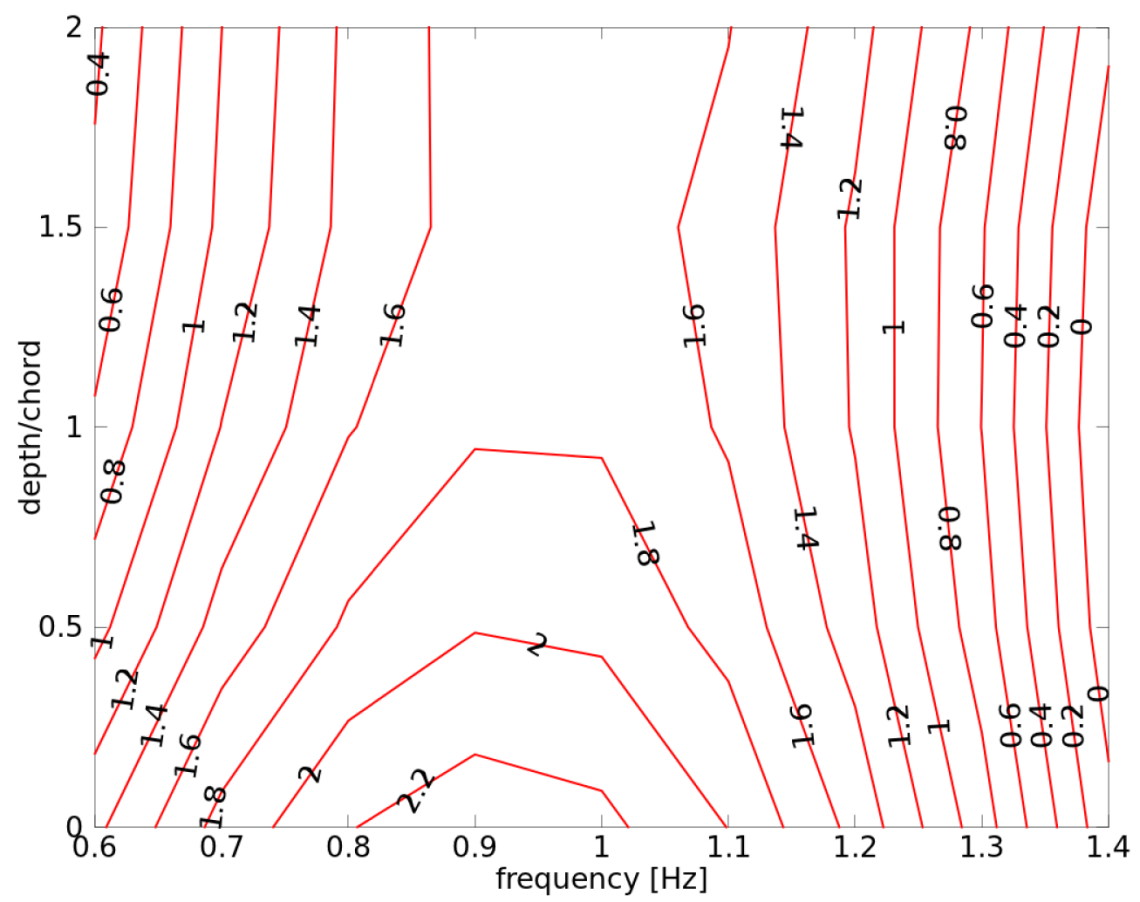

Figure 4: Contours of the thrust coefficient. 


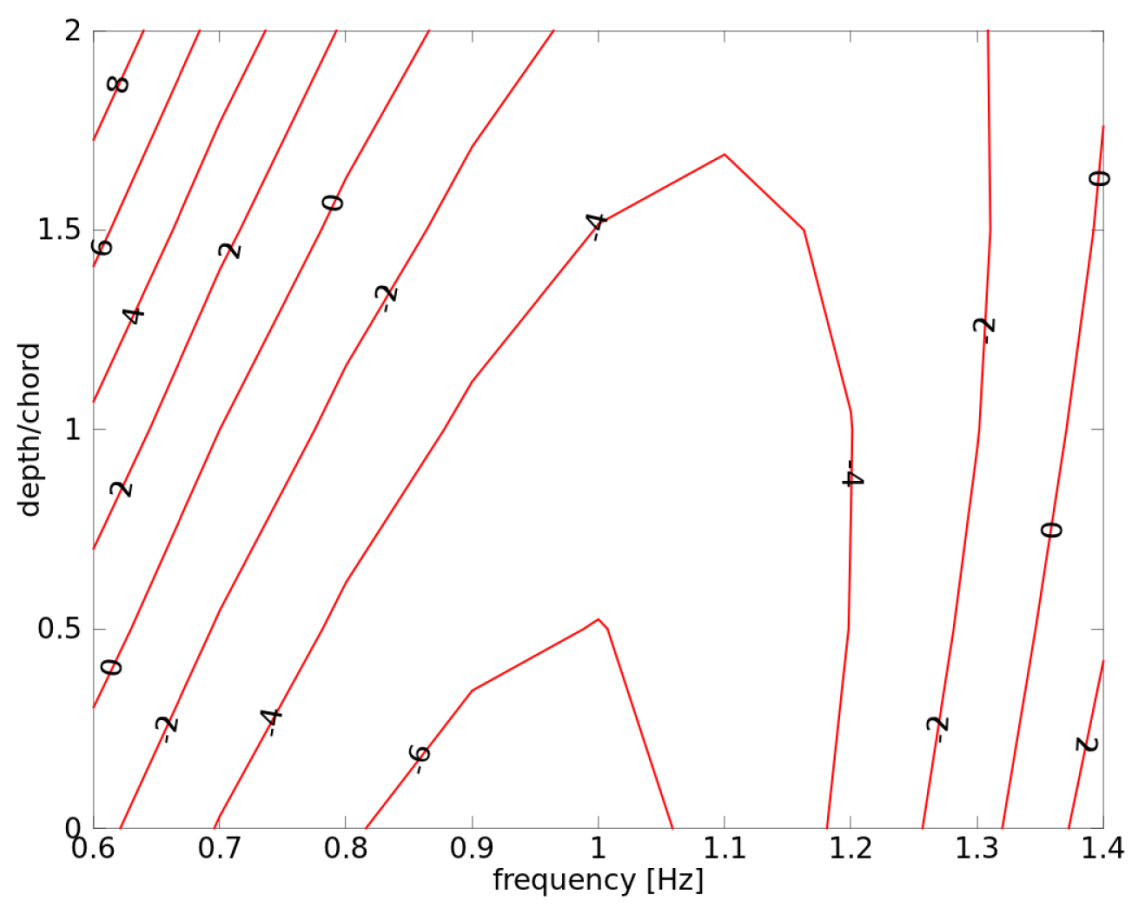

Figure 5: Contours of the lift coefficient.

thus an upstroke that demands less effort than in deep water. This is probably the explanation for the high efficiency.

A second way to interpret the data is to observe the equations of the regression surfaces:

$$
\left[\begin{array}{c}
c_{T}(f, d) \\
c_{L}(f, d) \\
\nu(f, d)
\end{array}\right]=A \cdot\left[\begin{array}{c}
1 \\
f \\
d \\
f^{2} \\
d^{2} \\
f d
\end{array}\right]
$$

with

$$
A=\left[\begin{array}{cccccc}
-5.80067 & 17.72861 & -1.31335 & -9.67383 & 0.22819 & 0.60267 \\
34.08770 & -86.96957 & 9.84139 & 46.28106 & 0.58526 & -9.01459 \\
-18.29956 & 37.16945 & 7.65269 & -17.15470 & -1.43399 & -5.86051
\end{array}\right]
$$

From this matrix we can see that the thrust and lift coefficients are mainly linearly and quadratically related to the frequency, and almost linearly related to 


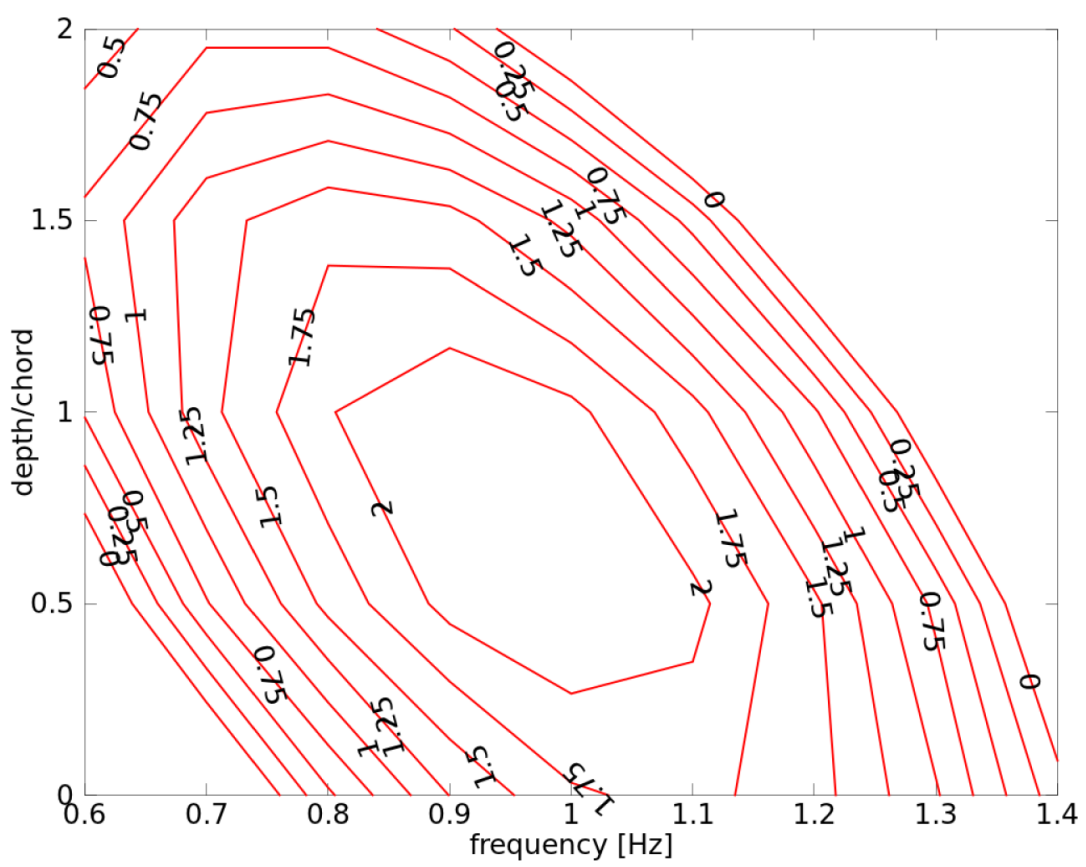

Figure 6: Contours of the efficiency.

the depth. $c_{L}$ is also related to the product $f d$. The efficiency is strongly related to all powers of the frequency and depth, except for $d^{2}$. This means that, as could be expected, the frequency has more influence on the performance than the depth, and that optimal conditions mainly depend on the former.

If the proximity of the free surface is beneficial for both the thrust and the efficiency, than why do marine mammals, who swim with a horizontal tail, do not prefer to swim near the surface? All bodies near the free surface have additional, surface making drag, and the gain in propulsive efficiency is probably not high enough to overcome this.

\section{Conclusion}

A series of experiments on a horizontal, heaving and passively-pitching hydrofoil, near the free surface was conducted for a range of heave frequencies and depths. Forces were measured, and the efficiency was calculated. Optimal conditions yield an average frequency and a rather close free surface.

\section{References}

[1] Webb, P.W., Hydrodynamics and energetics of fish propulsion. Bull Fish Res Bd Can, 190, pp. 1-158, 1971. 
[2] Breder, C.M., The locomotion of fishes. Zoologica, 4, pp. 159-297, 1926.

[3] Gillis, G.B., Undulatory locomotion in elongate aquatic vertebrates: Anguilliform swimming since sir james gray. American Zoologist, 36, pp. 656-665, 1996.

[4] Sfakiotakis, M., Lane, D. \& Davies, B., Review of fish swimming modes for aquatic locmotion. Journal of Oceanic Engineering, 42(2), pp. 273-252, 1999.

[5] Piziali, R.A., 2-d and 3-d oscillating wing aerodynamics for a range of angles of attack including stall. NASA Technical Memorandum 4632, 1994.

[6] Lai, J.C.S. \& Platzer, M.F., Jet characteristics of a plunging airfoil. AIAA Journal, 37(12), pp. 1529-1537, 1999.

[7] Anderson, J.M., Streitlien, K., Barrett, D.S. \& Triantafyllou, M.S., Oscillating foils of high propulsive efficiency. Journal of Fluid Mechanics, 360, pp. 41-72, 1998.

[8] Read, D.A., Hover, F.S. \& Triantafyllou, M.S., Forces on oscillating foils for propulsion and maneuvering. Journal of Fluids and Structures, 17, pp. 163$183,2003$.

[9] Hover, F.S., Haugsdal, O. \& Triantafyllou, M.S., Effect of angle of attack profiles in flapping foil propulsion. Journal of Fluids and Structures, 19, pp. 37-47, 2004.

[10] Lee, T. \& Gerontakos, P., Investigation of flow over an oscillating foil. Journal of Fluid Mechanics, 512, pp. 313-341, 2004.

[11] Heathcote, S., Wang, Z. \& Gursul, I., Effect of spanwise flexibility on flapping wing propulsion. Journal of Fluids and Structures, 24, pp. 183-199, 2008.

[12] Lauder, G.V., Anderson, E.J., Tangorra, J. \& Madden, P.G.A., Fish biorobotics: kinematics and hydrodynamics of self propulsion. Journal of Experimental Biology, 210, pp. 2767-2780, 2007.

[13] P. R. Bandyopadhyay, Beal D.N.N. \& Menozzi, A., Biorobotic insights into how animals swim. Journal of Experimental Biology, 211, pp. 206-214, 2008.

[14] Schreck, S.J. \& Helin, H.F., Unsteady vortex dynamics and surface pressure topologies on a finite wing. Journal of Aircraft, 31(4), pp. 889-907, 1994.

[15] Mason, R.L., Gunst, R.F. \& Hess, J.L., Statistical Design and Analysis of Experiments, 2003. 